\title{
Optimized treatment with RF thermotherapy and immunotherapy combined with CyberKnife for advanced high-risk tumors: A clinical trial report
}

\author{
ZHIGAO JIANG $^{1 *}$, QINWEN WANG ${ }^{3 *}$, GUIQING YANG $^{2}$, XIAOXU LIU ${ }^{2}$, \\ DONGNING SUN ${ }^{2}$, SHANSHAN WANG ${ }^{1},{\text { YANG } \text { LI }^{1} \text { and YISHAN WANG }}^{1,2}$ \\ ${ }^{1}$ Binzhou Medical College; ${ }^{2}$ Center for Tumor Treatment, People's Liberation Army 107th Hospital, Yantai, Shandong 264003; \\ ${ }^{3}$ Department of Radiotherapy, 307 Hospital Affiliated to the Military Academy of Medical Sciences, Beijing 100071, P.R. China
}

Received May 21, 2013; Accepted October 08, 2013

DOI: $10.3892 /$ br.2014.218

\begin{abstract}
This study was conducted to evaluate the application value of optimized treatment with radiofrequency (RF) thermotherapy and immunotherapy combined with CyberKnife for advanced high-risk tumors. The database of 1,013 patients with 2,136 tumor lesions and 1,237 target areas who underwent treatment with CyberKnife between November, 2010 and November, 2012, was retrospectively reviewed. We randomly assigned 505 eligible patients (observation group) to RF thermotherapy and adoptive immunotherapy with cytokine-induced killer cells and the remaining 508 patients (control group) to no adjuvant treatment. The patients in the two groups were recorded on efficacy assessment according to imageological examination, World Health Organization criteria, Karnofsky performance status, or radioimmunoassay (RIA) detection. The effective rate of the observation group was $75.05 \%$, whereas that of the control group was $58.06 \%$ $(\mathrm{P}<0.05)$. The results revealed that $\mathrm{CyberKnife}$ combined with hyperthermia and biological therapy are highly effective in improving the local tumor control rate. Further analysis of the Karnofsky score and RIA detection confirmed that this type of combination therapy significantly improved the quality of life. The optimized treatment of RF thermotherapy and immunotherapy combined with CyberKnife may act synergistically in eliminating tumor cells, confirming the efficacy of this type of treatment for patients with advanced malignant tumors.
\end{abstract}

Correspondence to: Dr Yishan Wang, Center for Tumor Treatment, People's Liberation Army 107th Hospital, 7 Zhichu South Road, Yantai, Shandong 264003, P.R. China

E-mail: wangyishan288@163.com

${ }^{*}$ Contributed equally

Key words: optimized treatment, CyberKnife, hyperthermia, immunotherapy, advanced high-risk tumor

\section{Introduction}

The treatment of advanced tumors may be complicated. Tumor metastasis, invasion of important organs and patients with middle- to advanced stage at high risk, are considered to be surgical taboos. Chemotherapy is crucial in cancer treatment; however, certain patients cannot tolerate the adverse reactions, such as gastrointestinal discomfort, hepatic damage, bone marrow suppression and toxic effects on the urinary system (1). Thus, palliative treatment is applied clinically in the majority of cases. The growing tumor may directly invade local sensors and oppress the adjacent nerves, blood vessels and soft tissues, leading to edema, inflammatory pain and tumor compression syndrome, which add to the suffering of the patients. CyberKnife is a type of advanced instrument that converges multiple radiation beams to deliver a single, large dose of radiation to a discrete tumor target with high precision. Due to the advantage of precise biologically effective dose delivery for target localization, while minimizing irradiation of the surrounding normal tissue, CyberKnife has become a new choice for the treatment of advanced metastatic high-risk tumors (2). We use CyberKnife combined with local heating treatment and immunotherapy with autologous cytokine-induced killer (CIK) cells to effectively control or ablate the tumor target. The local symptoms were reduced and the in vivo environment was improved, ultimately achieving optimization of systemic and local conditions, control of tumor development and decrease of adverse reactions and complications.

\section{Materials and methods}

Patients. The medical records of 1,013 patients with advanced high-risk tumors who were treated at the Center for Tumor Treatment of the People's Liberation Army 107th Hospital between November, 2010 and November, 2012 were reviewed.

The inclusion criteria were as follows: i) pathological, computed tomography (CT) or magnetic resonance imaging (MRI) diagnosis confirmation of stage III-IV tumors according to the World Health Organization (WHO) clinical staging standards; ii) Karnofsky performance status (KPS) $\geq 50$; iii) postoperative recurrence, involvement of important func- 
tions and central organs, lymph node infiltration or multiple metastases, lack of surgical indications or intolerability to conventional radiation and chemotherapy; iv) patient informed consent for tumor detection and treatment.

The exclusion criteria were as follows: i) concurrent tuberculosis or lack of definitive diagnosis; ii) diagnosed with tumor of the cavity organs; iii) recently repeated radiotherapy or suspected radiation complications.

A total of 1,013 patients with 2,136 tumor lesions and 1,237 target areas underwent CyberKnife therapy. We randomly assigned 505 eligible patients (observation group) to RF thermotherapy and adoptive immunotherapy with CIK cells and the remaining 508 patients (control group) to no adjuvant treatment. Detailed characteristics of the patients are presented in Table I.

This clinical trial of stereotactic body radiation therapy CyberKnife, radiofrequency (RF) thermotherapy and CIK cell-based immunotherapy was approved by the Institutional Ethics Committee and all the patients provided written informed consent.

\section{Research methods}

CyberKnife therapy. Following admission, an enhanced CT scan with a $1.25-\mathrm{mm}$ slice thickness was performed prior to CyberKnife treatment. If necessary, positron emission tomography-CT or MRI and digital subtraction angiography image fusion were also used to accurately determine the target and 2-4 gold fiducial markers were implanted in lung, liver or pancreas under CT guidance. The next step was to delineate the clinical tumor volume according to the patient's tumor lesion number, lesion size, distribution and degree of risk, in order to determine the treatment planning and dose. The treatment was administered 1-6 times, once per week. In this clinical trial, the output dose rate of the stereotactic radiation wave operation platform (CyberKnife system; Accuray, Sunnyvale, CA, USA) was 400-600 cGy, approved by the US Food and Drug Administration for systemic treatment in August, 2001. The tumor dose line coverage was $85-95 \%$. For patients with small single tumor lesions, the general dose was 1-3 F, whereas for patients with multiple tumor lesions it was 4-6 F. The total tumor dose was 18-70 Gy and the single dose 5-20 Gy.

$R F$ thermotherapy. The thermotherapy in this research was applied with an in-fresh-field thermotherapy machine (Jilin Morestep Medical Equipment Co., Ltd., Changchun, Jilin, China). Two sets of high frequency source were deployed to an operating frequency of $30.32 \pm 1.5$ and $40.68 \pm 1.5 \mathrm{MHz}$. The output power was $1100 \mathrm{~W}$, with a measurement precision of $\pm 0.1^{\circ} \mathrm{C}$.

The thermocouples were placed through the puncture needle into the splanchnocoele to collect the temperature of three different measuring points, while two different temperatures of distrinct measuring points in the rectum and the external auditory canal were monitored. The highest, lowest and average temperatures were measured and recorded. Among the measured temperatures, the average temperature of the three measuring points of the splanchnocoele was identified as tumor T90, whereas the temperature of the external auditory canal was used as the reference temperature of the central nervous system. The rectal temperature was controlled to $1^{\circ} \mathrm{C}$ lower compared to the heating area (splanchnocoele).
Table I. Characteristics of 1,013 patients receiving CyberKnife or optimized treatment.

\begin{tabular}{|c|c|c|}
\hline Characteristics & $\begin{array}{l}\text { Observation } \\
\text { group }\end{array}$ & $\begin{array}{l}\text { Control } \\
\text { group }\end{array}$ \\
\hline \multicolumn{3}{|l|}{ Age (years) } \\
\hline Median & 53.8 & 51.9 \\
\hline Range & $18-79$ & $17-81$ \\
\hline \multicolumn{3}{|l|}{ Gender } \\
\hline Male & 331 & 343 \\
\hline Female & 174 & 165 \\
\hline \multicolumn{3}{|l|}{ Primary site } \\
\hline Head/neck & 130 & 144 \\
\hline Breast & 136 & 127 \\
\hline Abdomen & 121 & 132 \\
\hline Vertebral metastasis & 118 & 105 \\
\hline Maximum volume & $15 \times 9.5 \times 18 \mathrm{~cm}^{3}$ & $14 \times 8.9 \times 17 \mathrm{~cm}^{3}$ \\
\hline Minimum volume & $1 \times 0.5 \times 0.5 \mathrm{~cm}^{3}$ & $0.9 \times 0.5 \times 0.6 \mathrm{~cm}^{3}$ \\
\hline \multicolumn{3}{|l|}{$\begin{array}{l}\text { Maximum } \\
\text { number of lesions }\end{array}$} \\
\hline Liver & 14 & 12 \\
\hline Lungs & 15 & 12 \\
\hline $\begin{array}{l}\text { Central nervous system } \\
\text { (longest diameter) }\end{array}$ & 10 & 14 \\
\hline
\end{tabular}

The patients underwent a thermal therapy 3 times/week with the dose amounting to $40^{\circ} \mathrm{C} / \mathrm{min}$ for 2 weeks.

Immunotherapy. Peripheral blood $(50 \mathrm{ml})$ was obtained from each eligible patient and subjected to Ficoll-Hypaque density centrifugation. The monocytes were cultured in 6-well plates at $37^{\circ} \mathrm{C}$ in RPMI-1640 medium supplemented with $10 \%$ heat-inactivated fetal bovine serum, $100 \mathrm{U} / \mathrm{ml}$ penicillin and $100 \mathrm{lg} / \mathrm{ml}$ streptomycin for $24 \mathrm{~h}$. The mouse anti-human CD3 monoclonal antibody (OKT3; R\&D Systems, Minneapolis, $\mathrm{MN}$, USA) (50 ng/ml) and interleukin-2 (Beijing Sihuan Biopharmaceutical Co. Ltd, Beijing, China) $(1,000 \mathrm{U} / \mathrm{ml})$ were added to the culture media. CIK cells were cultured in vitro and expanded $>1,000$ times in 12 days. The infused cells, that had undergone strict quality control, were $>20$ billion, among which the proportion of active cells was $>99.5 \%$.

Evaluation and statistical analysis. The median follow-up period was 15 months (range, 5-24 months) and included regular subsequent re-examination by MRI or CT, Karnofsky score, radioimmunoassay (RIA) detection and WHO objective criteria of curative effect (3). Complete remission (CR) was defined as symptoms and physical signs totally disappearing for 4 weeks; partial remission (PR) was defined as tumor volume shrinkage of $>50 \%$ for $\geq 4$ weeks; stable disease (SD) was defined as no significant change observable for $\geq 4$ weeks, with a tumor volume increase of $\leq 25$ or shrinkage of $\geq 50 \%$; progressive disease (PD) was defined as new lesions appearing or the original lesion increasing $>25 \%$ in size. The total effective rate was calculated with the following equation: $(\mathrm{CR}+\mathrm{PR}) /$ total cases $\times 100 \%$. SPSS software, version 13.0 
A

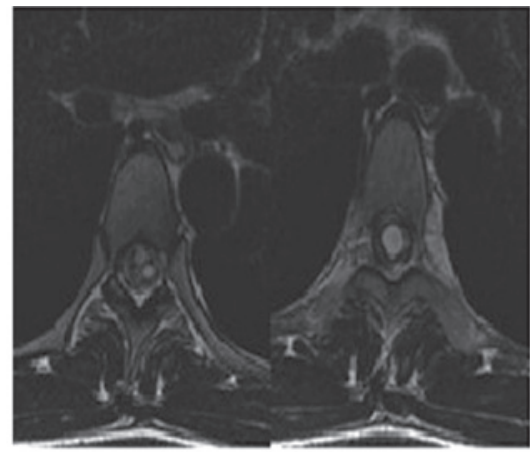

C

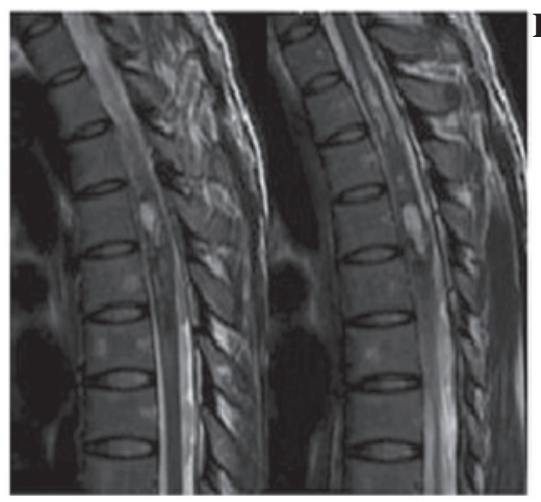

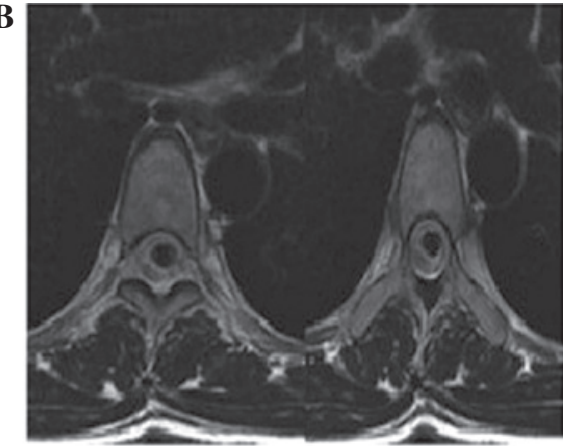

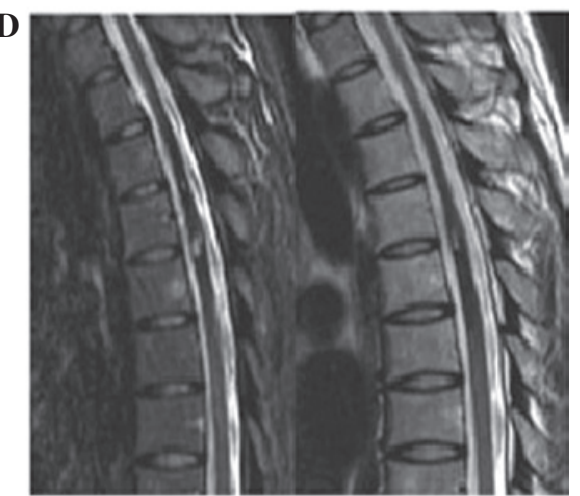

Figure 1. Chest intramedullary hemangioma $(14 \mathrm{~cm})$. (A) Computed tomography (CT) prior to CyberKnife therapy (2011-01-30). (B) CT after CyberKnife therapy (2011 04 30). (C) Magnetic resonance imaging (MRI) prior to CyberKnife therapy (2011-01-30). (D) MRI after CyberKnife therapy (2011-04-30).

(SPSS Inc., chicago, IL, USA) was used for statistical analysis. The objective curative effects between two samples were analyzed using the Chi-square test. The comparison of the Karnofsky scores prior to and following treatment was performed using the Wilcoxon's test. The Karnofsky scores and RIA detection between the observation and control groups were compared with the independent-samples t-test. $\mathrm{P}<0.05$ was considered to indicate a statistically significant difference.

\section{Results}

Patients. The participants included 1,030 high-risk patients with middle- to advanced-stage cancer, with multiple lesions and metastatic disease. There were 8 cases of disease exacerbation-related mortality; furthermore, 9 patients were discharged due to their inability to assume and/or maintain the forced supine position for long periods of time. Finally, a total of 1,013 cases were enrolled in the study. Patients with advanced cancer showed a high rate of alleviation of symptoms following treatment (specific cases shown in Figs. 1-3).

Karnofsky performance status. A distinct improvement of the Karnofsky scores was observed, accompanied by alleviation of tumor oppression syndrome and improvement of the quality of life after treatment. The comparison of the Karnofsky scores prior to and after treatment was performed using the Wilcoxon's test $(\mathrm{P}<0.05)$. Compared to the control group, the improvement of the Karnofsky scores in the observation group was more significant $(\mathrm{P}<0.05)$. A radiographic examination revealed tumor shrinkage of different degrees, even disappearance in some cases, following treatment. The curative effects
Table II. Preliminary clinical data.

\begin{tabular}{lcc}
\hline Variables & $\begin{array}{c}\text { Observation } \\
\text { group }\end{array}$ & $\begin{array}{c}\text { Control } \\
\text { group }\end{array}$ \\
\hline $\begin{array}{l}\text { KPS (mean } \pm \text { SD) } \\
\text { Prior to treatment }\end{array}$ & $64.73 \pm 8.05$ & $65.00 \pm 7.00$ \\
$\quad$ After treatment & $82.36 \pm 10.32$ & $73.64 \pm 9.87$ \\
Efficacy assessment $(\%)$ & & \\
$\quad$ Radiographic observation & 79.01 & 62.00 \\
RIA detection & 64.00 & 41.09 \\
WHO criteria & 75.05 & 58.06 \\
\hline
\end{tabular}

KPS, Karnofsky performance status; RIA, radioimmunoassay; WHO, World Health Organization.

in the two groups were analyzed using the Chi-square test $(\mathrm{P}<0.05)$.

RIA detection. RIA revealed that the tumor markers of the patients exhibited a reversed trend. Compared to the control group, the effective rate, as determined by tumor markers, was higher in the observation group $(\mathrm{P}<0.05)$.

WHO criteria. According to the WHO objective criteria of curative effect, the effective rate of the observation group was $75.05 \%$, whereas that of the control group was $58.06 \%$, with a statistically significant difference $(\mathrm{P}<0.05)$. The preliminary clinical data are listed in Table II. 


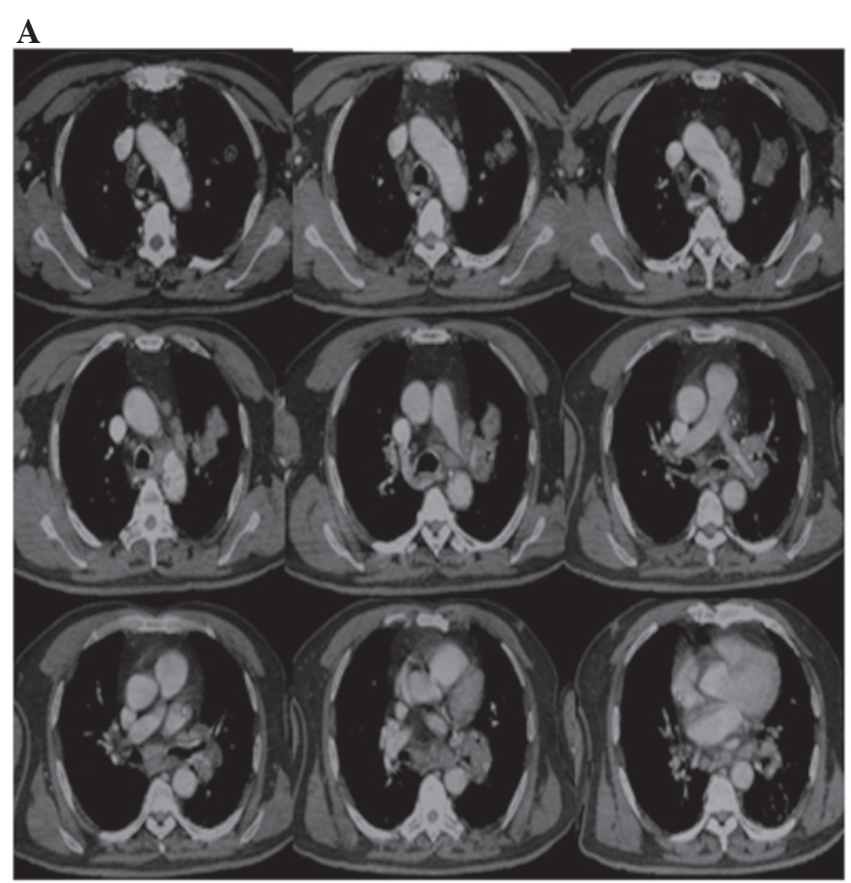

B

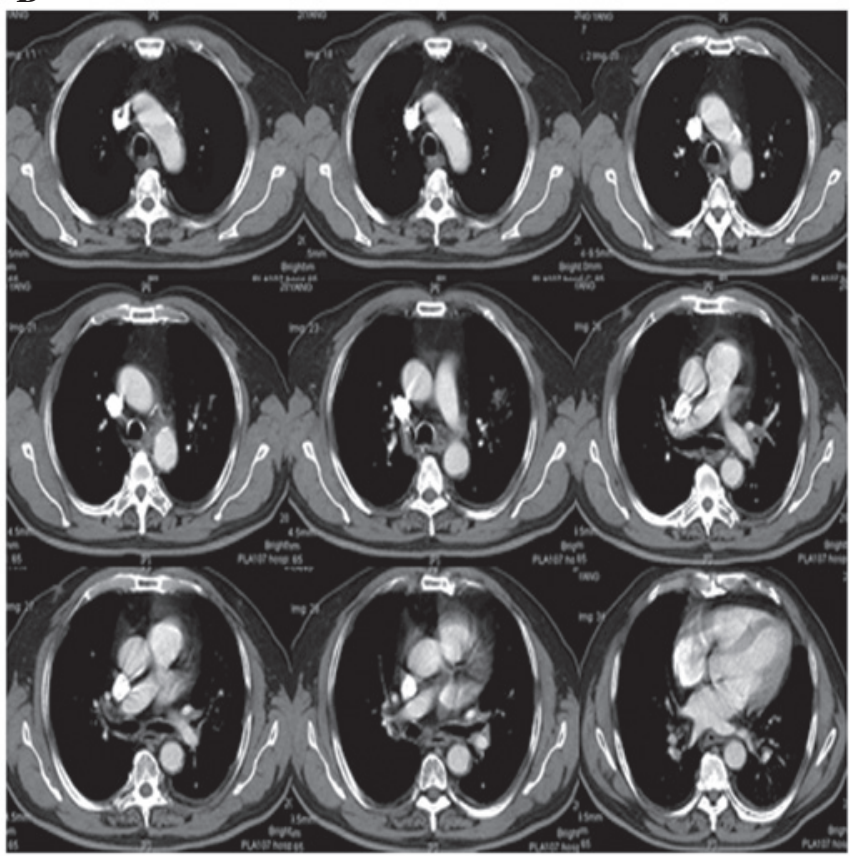

Figure 2. Lung cancer with mediastinal lymph node metastases (15 lesions). (A) Computed tomography (CT) prior to optimized treatment (2011-01-28) (B) CT after optimized treatment (2011-03-11).

\section{Discussion}

Surgical treatment may be challenging, since numerous patients with advanced high-risk tumors are unable to tolerate the adverse reactions of chemotherapy, surgical trauma and loss of blood. Furthermore, the damage of important organs along with central nervous system limitations compel clinicians to select alleviative treatment.

The precision and accuracy of CyberKnife treatment have greatly improved the curative rate, while a high-dose, lower

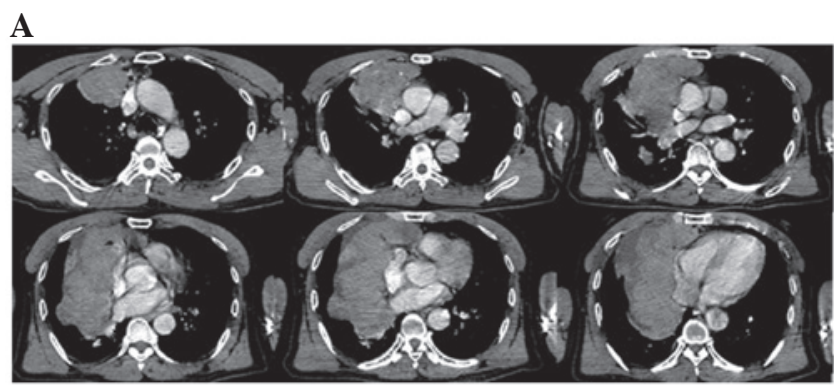

B

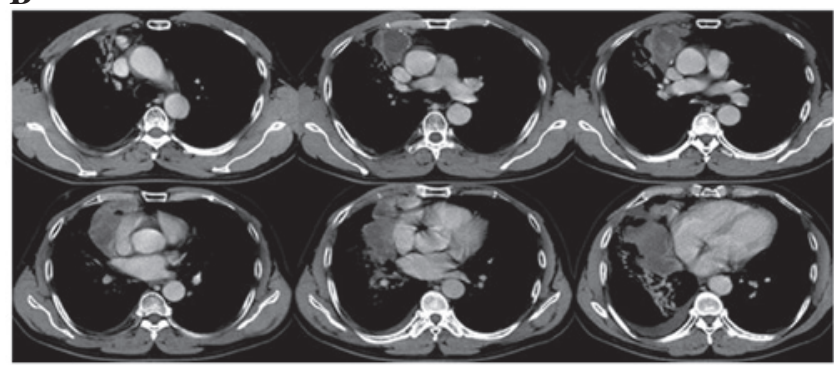

Figure 3. Sizeable thymoma $(15 \times 11 \times 18 \mathrm{cc})$. (A) Computed tomography (CT) prior to optimized treatment (2011-02-28). (B) CT after optimized treatment (2011 04 12).

treatment frequency treatment concept has been configured (4). As a result, short treatment time, non-toxic side effects and no hospital stay have been overemphasised in previous studies $(5,6)$. We recommend that the frequency, dosage and the number of CyberKnife treatments are determined according to the size and location of the tumor, the degree of risk and the previous treatments. However, given the accuracy and flexibility of the CyberKnife treatment, there should be emphasis on treatment individualization rather than shortening the treatment cycle. Although the condition of patients with multiple metastatic tumors may be timely controlled, oncolytic reaction and abnormal metabolism of bilirubin must be prevented during the early stages. Previous clinical trials reported that the hemogram was persistently normal during treatment with CyberKnife; however, the sudden decrease in granulocyte numbers after 2 weeks cannot be ignored, since it may lead to serious complications (7-8). Thus, it is advocated to keep the patients who undergo CyberKnife therapy with more than two treatment plans hospitalized with symptomatic treatment for $>2$ weeks, until the hemogram returns to normal. The overemphasis on the concept of non-toxic therapy should be avoided, considering the existence of serious adverse reactions on which attention must be focused.

With the image-tracking techniques, CyberKnife lays a solid foundation for target depiction through obtaining real three-dimensional images of tumors or vascular lesions. Thus far, the majority of radiation experts have been quoting or duplicating the traditional pattern of radiation biology and the prescribed dose based on the recommendations of Sinai Hospital, New York and the new biological pattern is not yet considered adequately sound. Furthermore, due to the limited basic testing on large samples, limited clinical research and significant disparity of treatment dosage for the same disease, there is yet no unified protocol among different countries regarding the dose of CyberKnife treatment. Furthermore, 
there is a lack of theoretical basis and practical experience on deducing the additional dose and optimizing dosage. Due to its accuracy and flexibility, CyberKnife therapy may alleviate the pain symptoms, relieve the compression syndrome, improve the quality of life and increase the chances of overcoming advanced multiple tumors $(2,7)$. In addition, CyberKnife exhibits significant radiosurgical value. In order to increase the indications of radiosurgery and reduce the related complications, the comprehensive treatment must be optimized and customised into an individualized treatment plan.

Thermotherapy is currently an important method for the treatment of middle- and advanced-stage malignant tumors. It was previously demonstrated that RF thermotherapy acts synergistically with chemotherapy and radiotherapy (8). By altering the pharmacokinetic parameters and increasing the regional blood flow of tumors, thermotherapy may enhance the cytotoxicity and improve the therapeutic response to anticancer drugs. Other evidence suggests that thermotherapy may help inhibit DNA repair by regulating the DNA polymerase; furthermore, the synthesis and secretion of vascular endothelial growth factor is restrained and the formation of new blood vessels is effectively reduced $(9,10)$. In addition, thermotherapy may enhance the cytotoxic effect of irradiation on S-phase cells and alleviate the toxicity reaction of normal tissue in selected cases (11). As a result, through increasing the blood supply of the surrounding normal tissue, thermotherapy enhances the biological effect of radiation (12).

Adoptive immunotherapy has been considered as the fourth kind of antitumor therapy (13). Previous in vitro and in vivo studies reported that immunotherapy is an effective method to lower the recurrence and metastatic rate of malignant tumors due to its high proliferative and cytolytic activity against various types of tumor cells $(14,15)$. Comprehensive immunotherapy treatments aimed at clearing micrometastatic cancer cells may provide highly flexible treatment combinations for cancer (16).

In our study, all the patients received the same postoperative care and the effects in the two groups were observed and evaluated. The effective rate of the observation group was $75.05 \%$, whereas that of the control group was $58.06 \%$, exhibiting a statistically significant difference $(\mathrm{P}<0.05)$. The result revealed that CyberKnife combined with hyperthermia and biological therapy is a regimen highly effective in improving the local tumor control rate. Further analysis of the Karnofsky scores and RIA detection confirmed that this type of combination therapy may significantly improve the quality of life, enhance the immunity of the patients and eliminate the remaining cancer cells on the basis of efficient local control.

Several scholars have investigated the separate curative effects of CyberKnife, RF thermotherapy and CIK cells in the treatment of middle- to late-stage malignant tumors. In this study, an optimal combination of these three approaches was first used in the clinical treatment of such patients. The analysis in this study allows the conclusion that the individual modalities included in this optimized treatment plan may act synergistically in eliminating tumor cells, confirming the efficacy of this treatment as a modern option for patients with advanced malignant tumors.

\section{Acknowledgements}

This study was supported by grants from the National Special Issue of the Ministry of Health of China (no. W2009BX042).

\section{References}

1. Oliai C, Lanciano R, Sprandio B, Yang J, Lamond J, Arrigo S, Good M, Mooreville M, Garber B and Brady LW: Stereotactic body radiation therapy for the primary treatment of localized prostate cancer. J Radiat Oncol 2: 63-70, 2013.

2. Wang YY, Wang YS, Liu T, Yang K, Yang GQ, Liu HC Wang SS and Yang JL: Efficacy study of CyberKnife stereotactic radiosurgery combined with CIK cell immunotherapy for advanced refractory lung cancer. Exp Ther Med 5: 453-456, 2013.

3. Sharma S, Ott J, Williams J and Dickow D: Dose calculation accuracy of the Monte Carlo algorithm for CyberKnife compared with other commercially available dose calculation algorithms. Med Dosim 36: 347-350, 2011.

4. Wang YS, Wang YY, Jiang P, Ma JJ, Qu Z, Wang XL, Li JT and Jia XF: Short-term outcomes of CyberKnife therapy for advanced high-risk tumors: A report of 160 cases. Exp Ther Med 3: 725-727, 2012.

5. Meng MB, Zaorsky NG, Jiang C, Tian LJ, Wang HH, Liu CL, Wang J, Tao Z, Sun Y, Wang J, Pang QS, Zhao LJ, Yuan ZY and Ping W: Radiotherapy and chemotherapy are associated with improved outcomes over surgery and chemotherapy in the management of limited-stage small cell esophageal carcinoma. Radiother Oncol 106: 317-322, 2013.

6. Wang X, Wang YY, Jiang P, Ma JJ, Qu Z, Liu HC, Wang SS and Wang YS: Clinical application of CyberKnife for high-risk central nervous system tumors: A clinical trial report of 60 cases. Exp Ther Med 3: 105-108, 2012.

7. Lin MC, Chen CM, Tseng HM, Xiao F and Young YH: A proposed method to comprehensively define outcomes in acoustic tumor patients undergoing CyberKnife management. Stereotact Funct Neurosurg 91: 177-185, 2013.

8. Al-Mamgani A, Rooij Pv, Tans L, Verduijn GM, Sewnaik A and de Jong RJ: A prospective evaluation of patient-reported quality-of-life after (chemo)radiation for oropharyngeal cancer: which patients are at risk of significant quality-of-life deterioration? Radiother Oncol 106: 359-363, 2013.

9. Ohguri T, Imada H, Narisada H, Yahara K, Morioka T, Nakano K, Miyaguni Y and Korogi Y: Systemic chemotherapy using paclitaxel and carboplatin plus regional hyperthermia and hyperbaric oxygen treatment for non-small cell lung cancer with multiple pulmonary metastases: preliminary results. Int J Hyperthermia 25: 160-167, 2009.

10. Shen H, Li XD, Wu CP, Yin YM, Wang RS and Shu YQ: The regimen of gemcitabine and cisplatin combined with radiofrequency hyperthermia for advanced non-small cell lung cancer: a phase II study. Int J Hyperthermia 27: 27-32, 2011.

11. He B, Wang X, Shi HS, Xiao WJ, Zhang J, Mu B, Mao YQ, Wang W and Wang YS: Quercetin liposome sensitizes colon carcinoma to thermotherapy and thermochemotherapy in mice models. Integr Cancer Ther 12: 264-270, 2013.

12. Abiko T, Kawamura M, Izumi Y, Oyama T, Saito Y and Kobayashi K: Prediction of anti-tumour effect of thermochemotherapy with in vitro thermochemosensitivity testing for non-small cell lung cancer. Int J Hyperthermia 23: 267-275, 2007.

13. Collins RH Jr, Shpilberg O, Drobyski WR, Porter DL, Giralt S, Champlin R, Goodman SA, Wolff SN, Hu W, Verfaillie C, List A, Dalton W, Ognoskie N, Chetrit A, Antin JH and Nemunaitis J: Donor leukocyte infusions in 140 patients with relapsed malignancy after allogeneic bone marrow transplantation. J Clin Oncol 15: 433-444, 1997.

14. Wang YF, Kunda PE, Lin JW, Wang H, Chen XM, Liu QL and Liu T: Cytokine-induced killer cells co-cultured with complete tumor antigen-loaded dendritic cells, have enhanced selective cytotoxicity on carboplatin-resistant retinoblastoma cells. Oncol Rep 29: 1841-1850, 2013.

15. Linn YC and Hui KM: Cytokine-induced killer cells: NK-like $\mathrm{T}$ cells with cytotolytic specificity against leukemia. Leuk Lymphoma 44: 1457-1462, 2003.

16. Wang J, He M, Shi W, Sha H, Feng J, Wang S and Wang Y: Inducible costimulator (ICOS) enhances the cytolytic activity of cytokine-induced killer cells against gallbladder cancer in vitro and in vivo. Cancer Invest 27: 244-250, 2009. 